\title{
Simple Chemical Stains for Feature-Rich Super-Resolution and Cleared-Tissue Microscopy
}

\author{
Chenyi Mao, ${ }^{1}$ Min Yen Lee, ${ }^{1}$ Jing-Ru Jhan, ${ }^{1}$ and Joshua C. Vaughan ${ }^{1,2,}$ \\ 1. Department of Chemistry, University of Washington, Seattle, Washington, USA. \\ 2. Department of Mechanical Engineering, University of Washington, Seattle, Washington, USA. \\ *Corresponding author: jcv2@uw.edu
}

Two of the most important developments in fluorescence microscopy over the past 1-2 decades are super-resolution microscopy, for imaging small features beneath the $\sim 250 \mathrm{~nm}$ diffraction limit of resolution of traditional light microscopy, and cleared tissue microscopy, for deep imaging of intact specimens free from the debilitating effects of scattering that typically limit imaging to $\leq 100 \mu \mathrm{m}$ thick tissues. With relatively widespread adoption of these methods by biomedical researchers, superresolution microscopy and cleared tissue microscopy have enabled many important discoveries in recent years. These methods also pose new challenges and opportunities for the fluorescent labels used with them.

We report, here, the development fluorescent analogs of classic histopathology stains and their application to super-resolution microscopy and cleared-tissue microscopy. The procedures use inexpensive, commercially available small molecules to covalently stain various chemical groups on proteins and carbohydrates and are able to reveal a wealth of feature-rich details. With suitable optimization of the conditions, the small molecules are able to penetrate tissues much more rapidly than antibodies and yield very bright stains. Furthermore, the labeling of chemical groups is relatively robust to sample degradation that occurs, for instance, due to heavy fixation or paraffin-embedding routinely used in tissue processing. We demonstrate the use of these stains via cleared-tissue and super-resolution microscopy of kidney, muscle, testis, brain, and intestine.

Among several popular methods for super-resolution microscopy, we focus on expansion microscopy (ExM) due to its strong performance with 3D, multi-channel imaging of thin or thick specimens using widely available commercial instruments [1,2], although the stains we report here should work for other super-resolution techniques. In ExM, a swellable polymer hydrogel is used to expand a fixed specimen with surprisingly low distortion such that features closer than the $\sim 250 \mathrm{~nm}$ diffraction limit are resolvable in the expanded state. For cleared tissue microscopy, we have focused on ethyl-cinnamate (ECi) based tissue clearing due to its simplicity, although the labels should also work with other clearing methods. At its most basic level, ECi clearing is able to minimize refractive index inhomogeneities in samples infused with ECi due to the fact that the refractive index of ECi is close to that of dehydrated protein [3].

Our basic experimental approach is to stain abundant chemical groups on tissues with reactive dyes. For instance, primary amine groups are abundant on proteins in cells and tissues and are labeled with aminereactive dyes such as N-hydroxy succinimidyl ester (NHS ester). In a second example, we have used a two-step procedure to label carbohydrates on cells such as are enriched at the boundaries of cells, on basement membranes, and on a range of other structures. The two-step procedure consists of oxidation of the specimen so that vicinal diols on sugars are converted to aldehyde groups followed by a second step in which aldehydes on the sample are reacted covalently with hydrazide-functionalized fluorophores. Amine-reactive and aldehyde-reactive fluorophores are popular, commercially-available reagents and are available with a wide range of spectral properties (i.e., absorption and emission 
spectra). For expanded specimens, stains can be performed either before or after expansion, $[1,2,4]$ while ECi-cleared specimens are stained prior to clearing.

Figure 1 shows results that use a combination of labeling of carbohydrates and amines, together with traditional DNA stains, on a range of tissue specimens. The stains reveal a wealth of details by virtue of shape and contrast. We believe these tools will be broadly useful in fluorescence microscopy either alone or in combination with specific labeling of proteins (e.g. using antibodies) or nucleic acids (using fluorescence in situ hybridization). While it is rare to use nonspecific labeling in fluorescence, similar approaches are heavily used in histopathology with non-fluorescent colorimetric stains (not, generally, suited to 3D imaging) and electron microscopy which is typically limited to imaging of very thin sections in two dimensions [5].

\section{References}

[1] Chen et al., Science 347 (2015), p. 543.

[2] Chozinski et al., Nat. Methods 13 (2016), p. 485.

[3] Klingberg et al., J. Am. Soc. Nephrol. 28 (2017), p. 452.

[4] Ku et al., Nat. Biotechnol. 34 (2016), p. 973.

[5] The authors would like to acknowledge supported from the University of Washington, NIDDK Diabetic Complications Consortium grant DK115255, and NIH grant R01 MH 115767.
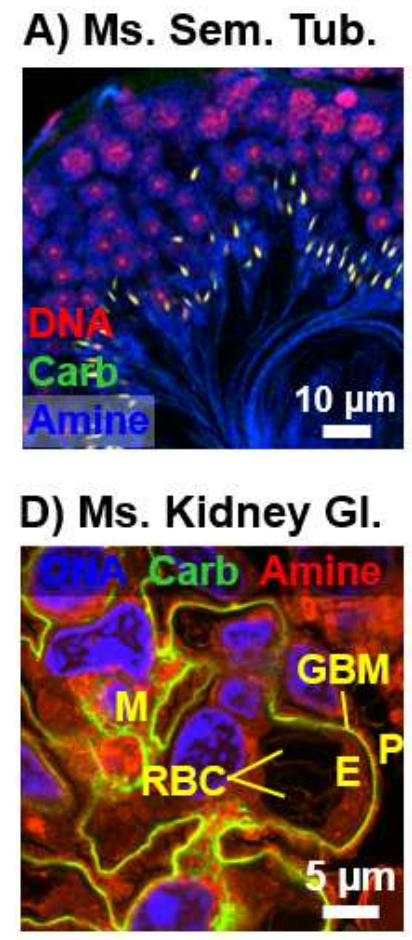
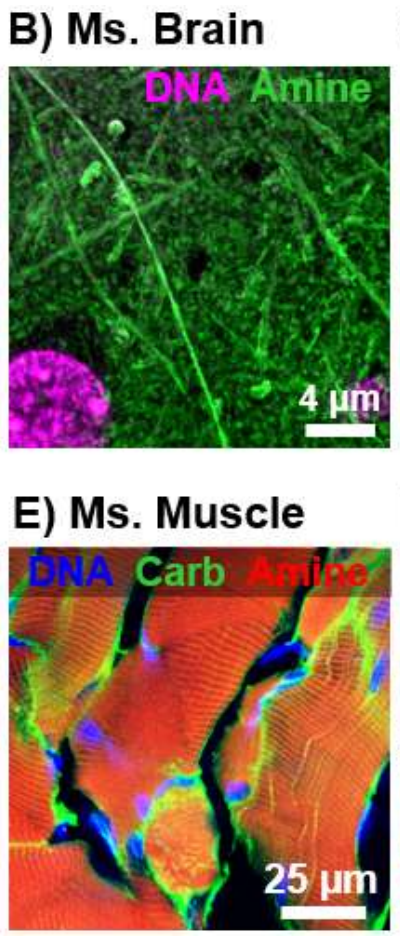

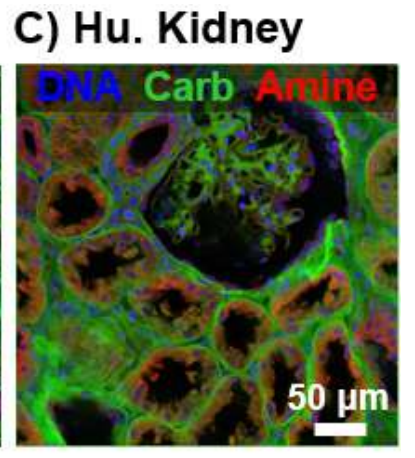

F) Ms. Intestine

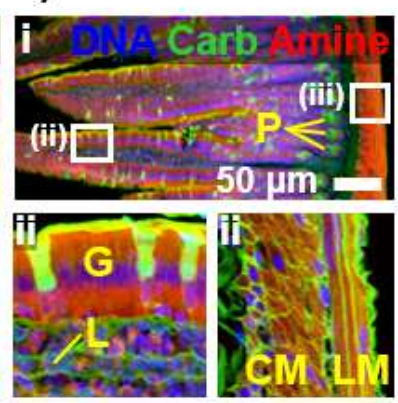

Figure 1. Small-molecule labeling of diverse tissues that have been cleared or expanded and stained for amines, carbohydrates, and DNA (with Hoechst) as indicated. A) Expanded mouse seminiferous tubule showing sperm developmental stages, with sperm heads in yellow (carbohydrates and DNA) and their blue tails curling toward the tubule lumen. B) Expanded mouse brain showing numerous features including myelin-rich axonal projections. C) Expanded human kidney tissue showing renal tubules and a glomerulus. D) Expanded mouse kidney glomerulus. The carbohydrate channel cleanly highlights the glomerular basement membrane (GBM) and the mesangial matrix (M). E) ECi-cleared mouse abdominal revealing periodic sarcomere z-bands and cellular boundaries. F) ECi-cleared mouse intestine. Intestinal villi are intensely stained along their surface by carbohydrates along with Paneth cells (P), goblet cells $(\mathrm{G})$, lymphatic capillaries (L), and cell boundaries of circular muscle (CM) and longitudinal muscle (LM). 